\title{
Wet Adhesion of Graphene
}

Wei Gao, K.M. Liechti and Rui Huang

Department of Aerospace Engineering and Engineering Mechanics, University of Texas, Austin, TX 78712

\begin{abstract}
Interfacial adhesion between graphene and various substrate materials is essential for practical applications of graphene. To date, most of the studies on adhesion of graphene have assumed dry adhesion of van der Waals type. In this paper, we conduct molecular dynamics simulations to study the traction-separation behaviors for wet adhesion of graphene on amorphous silicon oxide covered by a thin layer of water. Three stages of the traction-separation relations are identified and they are analyzed by simple,approximate continuum models. The work of separation is found to be close to the theoretical value dictated by the interaction potential between graphene and water. The maximum traction is found to be set by the critical stress for cavitation at the water/graphene interface. With morphological evolution of water from cavitation to capillary bridging, the range of interaction extends to about $3 \mathrm{~nm}$ before complete separation of graphene. Compared tovan der Waals interactionsfor dry adhesion of graphene, the work of separation for wet adhesion is smaller, the maximum traction is lower, but the interaction rangeis longer. It is noted that the properties of wet adhesion depend sensitively on the graphene-water interactions, which may vary considerably from hydrophobic to hydrophilic interactions.
\end{abstract}




\section{Introduction}

As an extremely thin crystal membrane, graphene has been studied extensively over the last decade for its physical properties and potential applications. In particular, the interfacial adhesion between graphene and various substrate materials is essential for the manufacture and integration of graphene [1-4]. To date, most of the studies on adhesion of graphene have assumed dry adhesion of van der Waals type [5-9]. However, measurements of the adhesion energy of graphene are often performed in ambient conditions[10-15], not in high vacuum. The results are likely influenced by the presence of water at the interfaces, depending on relative humidity of the environment. In the case of wet-transferred graphene $[1,15]$, both the graphene membrane and its substrate are directly exposed to liquid water during fabrication. A recent experimental studyreported ultra-long-range interactions between wet-transferred graphene and a silicon substrate [15], calling for further studies on other mechanisms of interfacial adhesion of graphene. This paper presents the first study on wet adhesion of graphene. We consider a relatively simple system with a pristine graphene monolayer on an amorphous silicon oxide (a$\mathrm{SiO}_{2}$ ) substrate, where a thin layer of water is sandwiched in between (see Fig. 1).

Previous studies [16-18] have shown that the silanol groups on $\mathrm{SiO}_{2}$ surfaces are sensitive to the adsorption of water molecules $\left(\mathrm{H}_{2} \mathrm{O}\right)$. Lee et al. [19] showed that water could diffuse between monolayer graphene and $\mathrm{SiO}_{2}$ substrates under high humidity conditions. The water diffusion results in the formation of a double ice-like water layer, which is quite stable, even under ambient conditions. Additional highly mobile and volatile liquid phase water can further diffuse between graphene and the ice-like layer on the $\mathrm{SiO}_{2}$ substrate. On the other hand,graphene is generally considered to be hydrophobic, with a contact angle of $\sim 90^{\circ}$ [20-24], although some recent studies have found that the contact angle of water on graphene could vary 
over a wide range, depending on airborne contamination [25, 26], defects [21], roughness [27], and the substrate on which it has been deposited [28, 29]. In principle, the contact angle can be related to the interactions between graphene and water. In this paper we adopt a model assuming hydrophobic interactions with a contact angle of $\sim 90^{\circ}$ for graphene.Molecular dynamics (MD)simulations are conducted to investigate the separation process with a sequence of morphological transitions of water between graphene anda- $\mathrm{SiO}_{2}$ substrate. Approximate continuum analyses are developed to help understand the MD results.Section 2 presents the model and method for MD simulations. The results are discussed in Section 3, followed by a summary in Section 4.

\section{MD model and method}

All MD simulations in this study are performed with LAMMPS [30] in NVT ensemble at 300Kusing the Nose-Hoover thermostat with an integration time step of $1 \mathrm{fs}$. As shown in Fig. 1, the MD model includes a monolayer graphene sheet and an a-SiO 2 substrate. Initially, a box of water molecules is placed between a-SiO 2 and graphene (Fig. 1a). The system is then relaxed at300 K, while two edges of the graphene (colored in pink) are fixed. During relaxation, the water molecules spread out on the surface of $\mathrm{a}-\mathrm{SiO}_{2}$ and the substrate is pulled up towards graphene. After a sufficiently long time, the system reaches an equilibrium state, with a continuous water film between graphene and $\mathrm{a}-\mathrm{SiO}_{2}$, as shown in Fig. 1b. Periodic boundary conditions are applied in all three directions. The in-plane dimensions of the periodic box are set by the graphene sheet in itsground state (about $10 \mathrm{~nm}$ by $10 \mathrm{~nm}$ ), while the a- $\mathrm{SiO}_{2}$ substrate is subjected toa small biaxial strain $(\sim 0.3 \%)$ to accommodate themismatch with graphene. The a$\mathrm{SiO}_{2}$ substrate is about $2 \mathrm{~nm}$ thick, and the thickness of the water film is varied from 1 to $4 \mathrm{~nm}$ 
by changing the number of water molecules; this thickness range is chosen to illustrate three different behaviors. The height of the periodic box is much larger $(40 \mathrm{~nm})$ to prevent interactions between periodic images in the thickness direction.
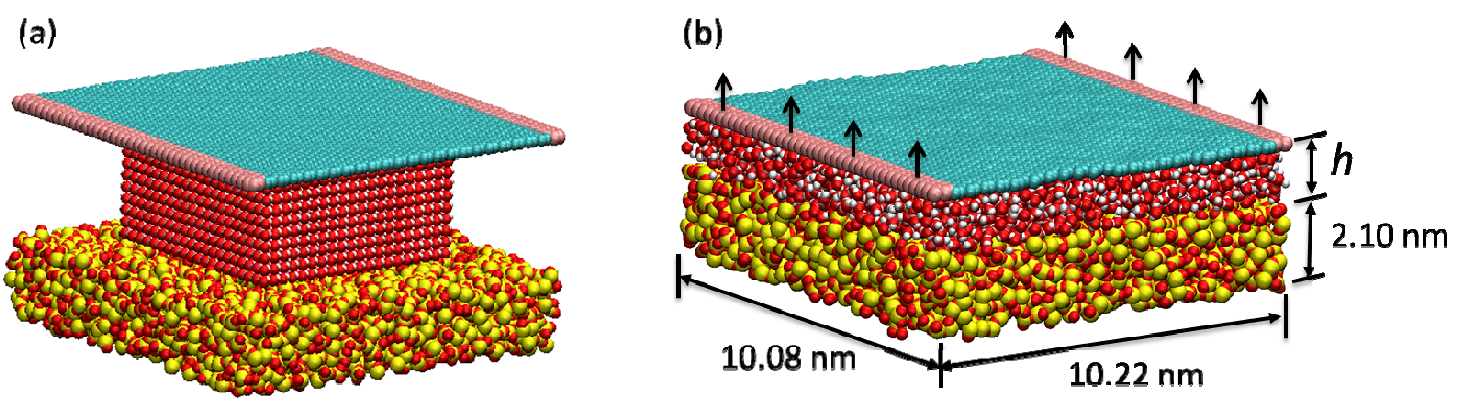

Figure 1: (a) Initial model with a box of water molecules; (b) Relaxed model with a continuous water film between a graphene monolayer andan $\mathrm{a}-\mathrm{SiO}_{2}$ substrate. The atoms are colored as $\mathrm{Si}$ (yellow), $\mathrm{O}$ (red), $\mathrm{H}$ (white), and $\mathrm{C}$ (green and pink). The pink $\mathrm{C}$ atoms are fixed during relaxation. During separation, the pink $\mathrm{C}$ atoms are pulled upwards with increasing displacement, while the atoms in the lower half of the a$\mathrm{SiO}_{2}$ substrate are fixed.

Simulations of the separation processes are conducted by increasing the prescribed displacement in the normaldirection for the carbon (C) atoms alongthe two clamped edges of the graphene, while the atoms in the lower half of the $\mathrm{a}_{-} \mathrm{SiO}_{2}$ substrateare fixed. The displacement is applied stepwise, with an increaseof $0.1 \AA$ at each step followed by relaxation for $1 \mathrm{~ns}\left(10^{6}\right.$ time steps). At each separation displacement, we compute the total interaction force between graphene and the substrate (including water), with which the average traction is obtained as a function of the displacement:

$$
\sigma(d)=\frac{\langle F(d)\rangle_{t}}{A}
$$

where $A$ is the area of the undeformed graphene sheet, $d$ is theprescribed edge displacement, and $\langle F(d)\rangle_{t}$ denotes the time average of the total force as a function of $d$. Using this molecular 
model, we study the traction-separation relation for wet adhesion of graphene and associated mechanisms.

The MD simulationsemploy a set of empirical force fields. The second-generation reactive empirical bond-order potential (REBO) is used to describe the $\mathrm{C}-\mathrm{C}$ interactions in graphene [31]. Previous studies [32-36] have shown that the REBO potential provides reasonable predictions of the mechanical properties of monolayer graphene. For the a-SiO 2 substrate, we usethe Tersoff potential [37], with a parameter set developed by Munetoh et al. [38] for the Si-O systems. The Tersoff potential is computationally efficientand predicts accurate mechanical properties of $\mathrm{SiO}_{2}$ in comparison withab initio calculations and experimental data [38].The water molecules are described by the extended simple point charge (SPC/E) model [39],characterized by three point masses with the $\mathrm{O}-\mathrm{H}$ bond distance of $0.1 \mathrm{~nm}$ and $\mathrm{H}-\mathrm{O}-\mathrm{H}$ bond angle equal to $109.47^{\circ}$. The constraints on the bond length and bond angle within each water molecule are enforced through the SHAKE algorithm [40]. Theinteratomic potential energy for water is given by:

$$
U_{i j}^{\text {water }}=4 \varepsilon_{i j}\left[\left(\frac{\delta_{i j}}{r_{i j}}\right)^{12}-\left(\frac{\delta_{i j}}{r_{i j}}\right)^{6}\right]+\frac{k_{e} q_{i} q_{j}}{r_{i j}},
$$

where $i$ and $j$ are indices for either $\mathrm{O}$ or $\mathrm{H}$ atoms of water, $r_{i j}$ is the interatomic distance, and $k_{e}$ is the electrostatic constant $\left(8.987 \times 10^{9} \mathrm{~N} \mathrm{~m}^{2} / \mathrm{C}^{2}\right)$. The potential energy includes two parts: the dispersion interactions represented by the Lennard-Jones (LJ) potential with two parameters $\left(\delta_{i j}\right.$ and $\varepsilon_{i j}$ ) for each pair and the electrostatic interactions between charges $q_{i}$ and $q_{j}$. According to the SPC/E model [39], the charges on $\mathrm{O}$ and $\mathrm{H}$ are -0.8476 and 0.4238 e, respectively. Meanwhile, only the $\mathrm{O}-\mathrm{O}$ interactions are included for the LJ potential with parameters 
$\delta_{\mathrm{OO}}=0.3166 \mathrm{~nm}$ and $\varepsilon_{\mathrm{oo}}=6.76 \mathrm{meV}$; the $\mathrm{O}-\mathrm{H}$ and $\mathrm{H}-\mathrm{H}$ dispersion interactions are much weaker and hence ignored. In MD simulations, the cutoff distancesfor the interactions areset to be $1 \mathrm{~nm}$ for the dispersion interactions and $1.2 \mathrm{~nm}$ for the electrostatic interactions, followingcommon practice [39]; the longer cut-off distance for the electrostatic interactions may be justified by noting the slower decay with increasing interatomic distance. The long-range electrostatic interactions are computed by using the particle-particle particle-mesh (PPPM) algorithm [41] as implemented in LAMMPS.

The mechanical property of water is essential for the present study. It is often assumed in theoretical analyses that water in its liquid phase is incompressible. However, measurements have found that the bulk modulus of liquid water is about 2.2 GPa[42].Using the SPC/E model for water, we perform MD simulationsof water under hydrostatic tension at $300 \mathrm{~K}$. In our calculation, a box of water molecules with periodic boundary conditions is subjected to an increasing volumetric strain (stepwise loading with displacement in all three directions, followed by relaxation at each step), and the virial stress is calculated as a function of the volumetric strain. As shown in Fig. 2,the average stress (hydrostatic tension) in water first increases with the volumetric strain, giving an initial bulk modulus of $2.07 \mathrm{GPa}$, close to the measuredvalue. Moreover, the hydrostatic tension increases nonlinearly andreaches a maximum of $142.2 \mathrm{MPa}$, beyond which the stress drops with increasing strain. The maximum hydrostatic tension is associated with onset of cavitation in waterand weakly depends on the size of the computational box. The critical stress for cavitation in water has been previously reported as ranging from 100 to $200 \mathrm{MPa}$ at room temperature [43-45]. Therefore, the SPC/E model captures the mechanical behavior of water reasonably well. 


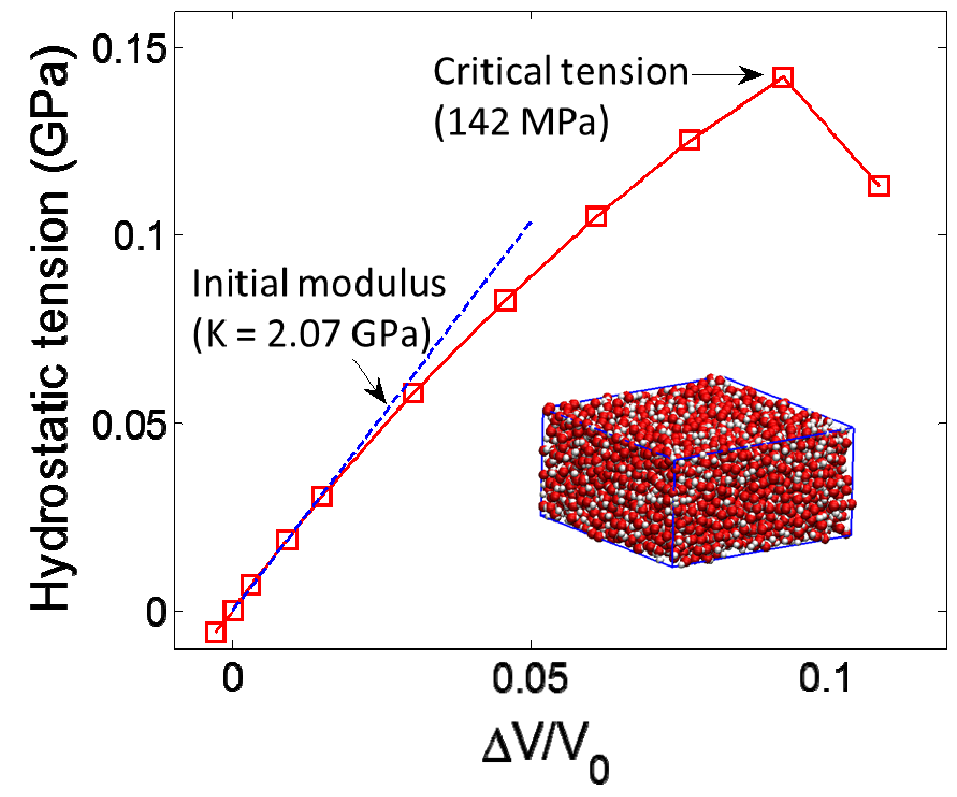

Figure 2: Nonlinear hydrostatic stress-strain relation of bulk water obtained from MD simulations using the SPC/E model. Inset shows the box of water molecules subjected to hydrostatic tension. The dashed line shows the linear approximation with an initial bulk modulus of $2.07 \mathrm{GPa}$ for small strains. The maximum tension is associated with the onset of cavitation in water.

In addition to the force fields for graphene, $\mathrm{a}-\mathrm{SiO}_{2}$ and water, interfacial force fields are needed to describe the interactions among them. First-principle calculations $[9,46]$ have shown that the interactions between graphene and water is dominated by dispersion interactions. Based on the contact angle and the water monomer binding energy on graphite, Werder et al. [47]calibrated a set of parameters for the LJ potential interacting between the oxygen atoms of water and the carbon atoms; the interactions between the hydrogen atoms of water and the carbon atoms were ignored. The $\mathrm{LJ}$ potential function takes the same form as the first term on the right-hand side of Eq. (2), with fitted parameters $\delta_{\mathrm{CO}}=0.319 \mathrm{~nm}$ and $\varepsilon_{\mathrm{CO}}=4.07 \mathrm{meV}$. These parameters lead to a theoretical adhesion energy of $60.0 \mathrm{~mJ} / \mathrm{m}^{2}$ between water and graphene, with an equilibrium separation of $0.274 \mathrm{~nm}$ [48]. Using this interaction potential along with theSPC/E model for water, we performed an MD simulation to determine the contact angle of a water 
dropleton graphene. As shown in Fig. 3, a box of water molecules relax to form a hemispherical droplet on graphene, with a contact angle of about $90^{\circ}$. Different contact angles may be obtained by modifying the fitted parameters ( $\delta_{\mathrm{CO}}$ and $\varepsilon_{\mathrm{CO}}$ ), which is left for future studies.
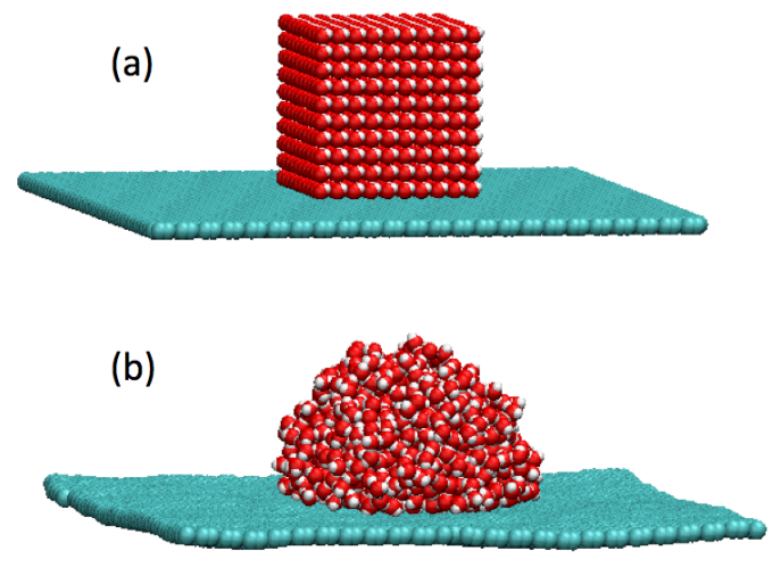

Figure 3: (a) A box of water molecules on graphene before relaxation, and (b) a hemispherical water droplet after relaxation at $300 \mathrm{~K}$, with a contact angle of about $90^{\circ}$.

The force field developed by Cruz-Chu et al. [49] is adopted for water- $\mathrm{SiO}_{2}$ interaction, which includes both electrostatic and van der Waals forces. Similar to the potential function in Eq. (2), the LJ parameters for the Si-O and O-O interactions are: $\delta_{\mathrm{SiO}}=0.347 \mathrm{~nm}, \varepsilon_{\mathrm{SiO}}=2.30$ $\mathrm{meV}, \delta_{\mathrm{OO}}=0.382 \mathrm{~nm}$, and $\varepsilon_{\mathrm{OO}}=1.63 \mathrm{meV}$, while the $\mathrm{H}$ atoms are ignored. The charges for the $\mathrm{Si}$ and $\mathrm{O}$ atoms in $\mathrm{SiO}_{2}$ are set to be 1 and -0.5 e, respectively.These parameters are chosen so that the surface of a- $\mathrm{SiO}_{2}$ is super-hydrophilic with a contact angleof almost $0^{\circ}$. However, as observed in previous studies $[49,50]$, the water- $\mathrm{SiO}_{2}$ interactions could bemuch more complicated, requiring empirical parameters to account for other surface characteristics of $\mathrm{SiO}_{2}$, which are beyond the scope of the present study.

Finally, the interaction between graphene and a-SiO 2 (without water) is assumed to be primarily due to van der Waals forces. According to the previous studies $[9,51,52]$, the range of the van der Waalsforces between graphene and $\mathrm{a}-\mathrm{SiO}_{2}$ is about $1 \mathrm{~nm}$, beyond which the 
interaction is negligible. For computational efficiency and based on the fact that the thickness of the water layer considered in this study is equal to or greater than $1 \mathrm{~nm}$, the van der Waalsforces between graphene and a-SiO${ }_{2}$ were not included in the $\mathrm{MD}$ simulations.

The a- $\mathrm{SiO}_{2}$ substrate in the $\mathrm{MD}$ simulations is obtained by a melt-quench-relaxation procedure, following the recipe of Litton and Garofalini[53]. We start by replicating the unit cell of alpha-quartz to construct a crystalline model of $\mathrm{SiO}_{2}$ and thenraisethe temperature to $10,000 \mathrm{~K}$ to melt the crystal and form liquidSiO ${ }_{2}$. Next, by quenching the liquidto $300 \mathrm{~K}$ through intermediate temperatures, we obtain a solid block of a- $\mathrm{SiO}_{2}$. In order to create a free surface for the a- $\mathrm{SiO}_{2}$ substrate, we immobilize the atoms at the bottom of the block and remove the periodic boundary condition in the normal direction of the surface. Finally, the system is relaxed consecutively at 2000,1000 , and $300 \mathrm{~K}(10 \mathrm{ps}$ at each temperature $)$. This procedure generates ana- $\mathrm{SiO}_{2}$ substrate with a macroscopically smooth surface. We note that more realistic surface structures of the a-SiO${ }_{2}$ substrate could be generated for $\mathrm{MD}$ simulations [54], including the surface roughness, but this is left for future studies.

\section{Results and discussion}

The traction-separation relations $(\sigma-d)$ obtained from MD simulations areshown in Fig. 4 (a-c) for three different water layer thicknesses. In each case, as the graphene is separated from the substrate, the water layer evolves from a continuous film to capillary bridges of different types, depending on the initial thickness of the water layer. The average traction first increases with increasing separation and then decreases. Eventually, the capillary bridge breaks upand the traction drops to zero. The area underneath the traction-separation curve gives the work of separation (per unit area), which may be different from the work of adhesion for the case of 'wet' 
adhesion due to possible hysteresis [55]. The work of separation is found to be $62.7,70.7$, and $66.9 \mathrm{~mJ} / \mathrm{m}^{2}$, respectively, for the three cases in Figure 4. These values are close to the theoretical value $\left(60.0 \mathrm{~mJ} / \mathrm{m}^{2}\right)$ dictated by the interaction potential between graphene and water. While the interaction potential itself is short-ranged (less than $1 \mathrm{~nm}$ with an equilibrium separation at 0.274 $\mathrm{nm}$ [48]), the morphological evolution of water in the MD simulations results in interactions over a relatively long range (up to $3 \mathrm{~nm}$ ). Meanwhile, the maximum traction is relatively low (about $90 \mathrm{MPa}$ ). Notably, the work of separation is considerably smaller than the adhesion energybetween graphene and dry $\mathrm{SiO}_{2}$ (i.e. no water) byvan der Waals interactions; the latter has been reported in the range of $0.1-0.45 \mathrm{~J} / \mathrm{m}^{2}$ [9-12]. This may be understood as a result of the hydrophobicity of graphene in the present model, which has a weaker graphene-water interactionthan the direct graphene- $\mathrm{SiO}_{2}$ interaction.On the other hand, the range of van der Waals interactions is shorter (less than $1 \mathrm{~nm}$ ) [9]. Therefore, the presence of a water layer could effectively increase the range of interactions between graphene and the substrate, but the range is still much shorter than that reported in [15] based on large-scale experiments. Other effects such as surface roughness may have to be considered in future studies in order to close the gap.

In all three cases of Fig. 4 (a-c), the traction-separation relations and the associated morphological evolution of water can be divided into three stages: (I) increasing traction with continuous water film (A to B), (II) decreasing traction withinterfacial cavitation (B to D), and (III) decreasing traction with capillary bridging (D to G), as illustrated schematically in Fig. 4d. While the traction-separation relations are similar in the three cases, the morphological evolution of water (especially cavitation and bridging) depends on the initial thickness of the water layer,as observed inthe MD simulations with three different thickness values (Fig. 4 a-c). In the following we discuss the three stages in details with associated continuum analyses. 

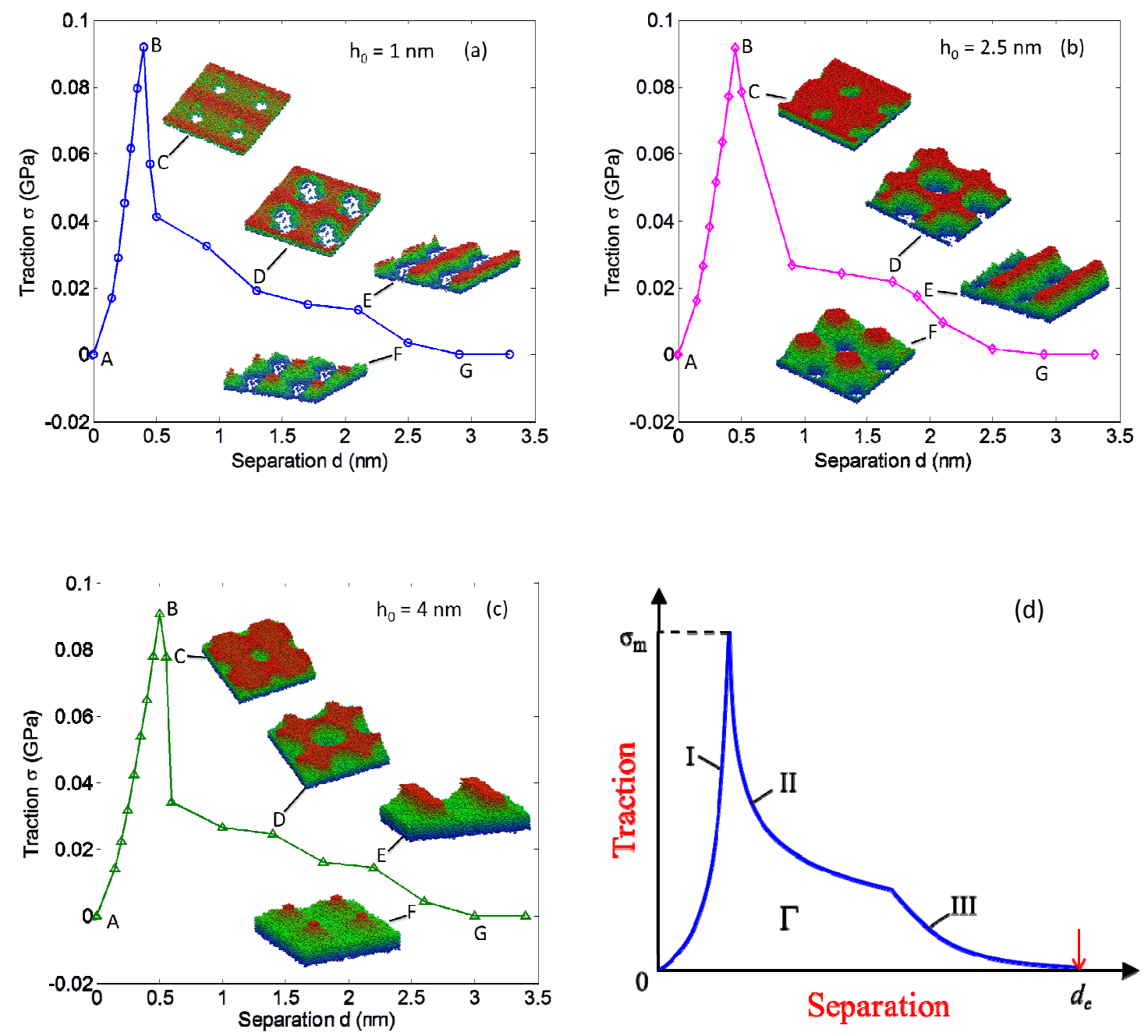

Figure 4: (a-c) Traction-separation relations for graphene on $\mathrm{a}_{-} \mathrm{SiO}_{2}$ with a water film of initial thickness equal to 1, 2.5, and $4 \mathrm{~nm}$, respectively. The insets are snapshots ( 2 by 2 periodic replication) from the MD simulations showing only the water molecules, with colors for the height contours. (d)A schematic illustration of the three stages in the traction-separation relation:(I) continuous water film (A-B), (II) cavitation (C-D), and (III) capillary bridging (E-F). Note the maximum traction $\sigma_{m}$, range of interaction $d_{c}$ and the work of separation $\Gamma$.

\subsection{Stage I: Continuous water film}

In the first stage of separation, the water remains a continuous film and undergoes a tensile deformation. Meanwhile, the graphene sheet deforms coherently as illustrated in Fig. 5a. The interaction between graphene and water pulls the water up and drags the graphene down, whereas the hydrophilic interaction between water and $\mathrm{a}-\mathrm{SiO}_{2}$ keeps the water film attached to 
the substrate. The behavior of this stage can be analyzed approximately by a simple continuum model.

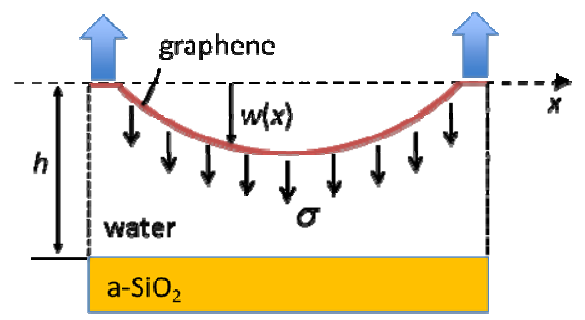

(a)

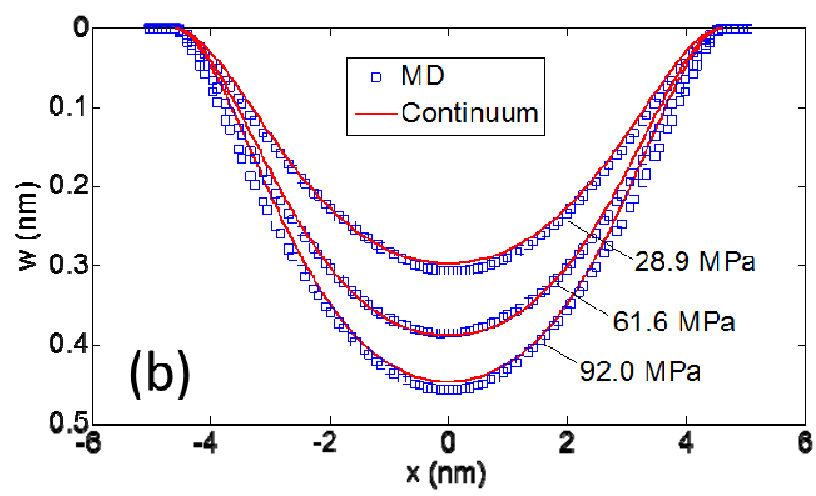

Figure 5: (a) Illustration of a continuum model for a graphene sheet on a continuous water film, subjected to a uniform traction $\sigma$; (b)Deflections of graphene under three tractions, comparingthe continuum analysis with MD simulations.

The graphene monolayer can be modeled approximately as a continuum plate [34] subject to a uniform normal traction $\sigma$ with two clamped edges(Fig. 5a). The total potential energy of grapheneis:

$$
\Pi=\frac{D L}{2} \int_{-L / 2}^{L / 2}\left(\frac{\partial^{2} w}{\partial x^{2}}\right)^{2} d x+\frac{E_{2 D} L}{2\left(1-v^{2}\right)} \int_{-L / 2}^{L / 2}\left[\frac{\partial u}{\partial x}+\frac{1}{2}\left(\frac{\partial w}{\partial x}\right)^{2}\right]^{2} d x-\sigma L \int_{-L / 2}^{L / 2} w d x
$$

where $L$ is the length of the square graphene sheet, $E_{2 \mathrm{D}}, v$ and $D$ are in-plane Young's modulus, Poisson's ratio and bending modulus of graphene, $w$ is theout-of-plane deflection and $u$ is the inplane displacement. The equilibrium deformation of graphene can be determined by minimizing the potential energy, leading to a set of nonlinear differential equations for $w(x)$ and $u(x)$. An approximate solution to the nonlinear problem takes the form[56]: 


$$
\begin{aligned}
& w(x)=\left(a_{1}+a_{2} x^{2}\right)\left(\frac{L^{2}}{4}-x^{2}\right)^{2} \\
& u(x)=x\left(b_{1}+b_{2} x^{2}\right)\left(\frac{L^{2}}{4}-x^{2}\right)
\end{aligned}
$$

where $a_{1}, a_{2}, b_{1}$ and $b_{2}$ are the parameters to be determined. Substituting (4) into (3), the total potential energy for graphene is obtained as a function of the four parameters, $\Pi\left(a_{1}, a_{2}, b_{1}, b_{2}\right)$. Minimizing the potential energy by setting $\frac{\partial \Pi}{\partial a_{i}}=0$ and $\frac{\partial \Pi}{\partial b_{i}}=0$ with $i=1$ and 2,we obtain four algebraic equations that can be solved to determine the four parameters, with whichwe obtain the deflection profile for each given traction $\sigma$.To compare with MD simulations, the elastic properties of graphene predicted by the REBO potential [34] areused: $E_{2 \mathrm{D}}=243 \mathrm{~N} / \mathrm{m}, v=0.397$, and $D=1.4 \mathrm{eV}$.Figure $5 \mathrm{~b}$ shows that the deflections of graphene predicted by the continuum analysis arein close agreement with the MD simulations.

Next, we consider deformation of the water film. With the deflection profile of graphene, the volume change of water can be calculated as:

$$
\Delta V=L^{2} d-L \int_{-L / 2}^{L / 2} w(x ; \sigma) d x
$$

On the other hand, the volume change of the water film can be related to the traction byits constitutive behavior:

$$
\Delta V=f(\sigma) V_{0}
$$

where $V_{0}=L^{2} h_{0}$ and $f(\sigma)$ is a nonlinear function for the hydrostatic stress-strain relation of the water film. Note that, by force balance, the hydrostatic tension in water equals the average 
traction acting on graphene; this is true under the condition that the interactions between graphene and a- $\mathrm{SiO}_{2}$ are negligible. By Eq. (6), the function $f(\sigma)$ can be obtained from MD simulations. It is found that $f(\sigma)$ depends on the initial thickness of the water film. As shown in Fig. 6(a), the stress-strain relation obtained from MD simulations is similar to the bulk water for a relatively thick water film $\left(h_{0}=4 \mathrm{~nm}\right)$, but becomes more compliant for thinner water films. This may be attributed to the interfacial compliance between graphene and water, which becomes more important for thinner water films. By fitting the hydrostatic stress-strain curvein Fig. 6a with a nonlinear function $f(\sigma)$ for each case and combining Eqs. (5)-(6), we obtain atraction-separation relation:

$$
d(\sigma)=f(\sigma) h_{0}+\frac{1}{L} \int_{-L / 2}^{L / 2} w(x ; \sigma) d x
$$

As shown in Fig. 6b,this relation compares closely with the MD simulations for the first stage up to the maximum traction ( $\sim 90 \mathrm{MPa}$ ). We note that the first term on the right-hand side of Eq. (7) depends on the initial thickness $\left(h_{0}\right)$ of the water film. For a relatively thick water film $\left(h_{0} \geq 4\right.$ $\mathrm{nm}), f(\sigma)$ is given by the constitutive behavior of bulk water, independent of $h_{0}$; hence the separation dincreases linearly with $h_{0}$. For a thinner water film $\left(h_{0}<4 \mathrm{~nm}\right)$, however, the dependence on the water thickness is more complicated since $f(\sigma)$ becomes thickness dependent. The second term on the right-hand side ofEq. (7) depends on the deformation of graphene, which also depends on the size of the graphene sheet $(L)$. In general, we expect the separation to increase when the water thickness or the graphene sheet size increases in the first stage of separation. 


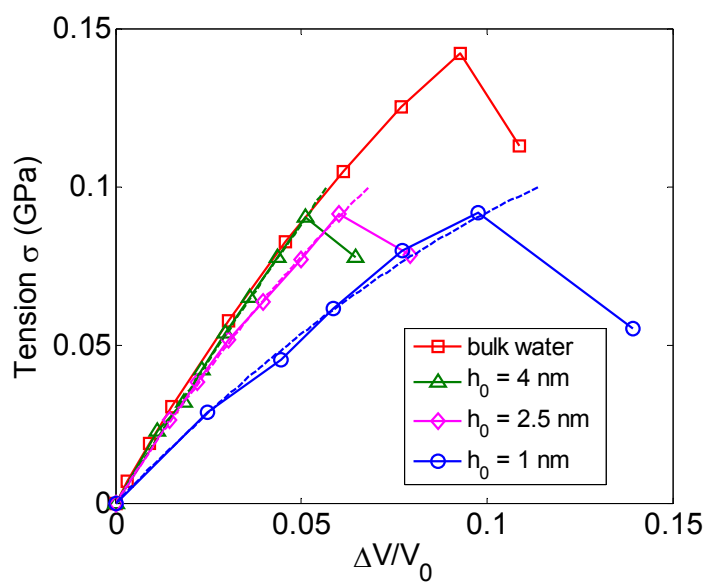

(a)

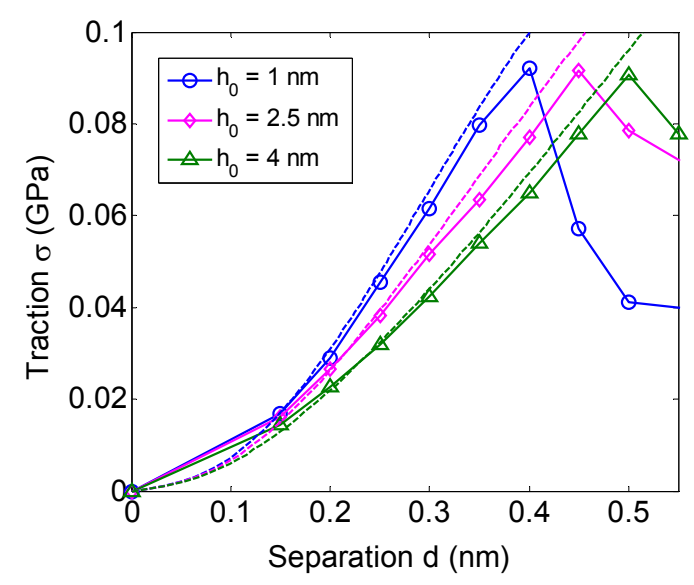

(b)

Figure 6: (a) Volumetric stress-strain relations of water films with different initial thicknesses $\left(h_{0}=1,2.5\right.$ and $4 \mathrm{~nm}$ ), in comparison with bulk water. The dashed lines show the fittingsby Eq. (6) with a nonlinear function $f(\sigma)$ for each case. (b) Traction-separation curves for Stage I, comparing the continuum analysis (dashed lines) with the MD simulations (symbols).

The end of Stage I is marked by the maximum traction $\left(\sigma_{m} \sim 90 \mathrm{MPa}\right)$ in the tractionseparation relation. Similar to the hydrostatic stress-strain behavior of bulk water in Fig. 2, the maximum traction is associated with cavitation of water.However, unlike the bulk water, cavitation of the water film occurs exclusively at the interface between water and graphene, due to relatively weak interactions across interface in the present model. As a result,the critical stress for cavitation is considerably lower for the water film than for bulk water (see Fig. 6a). Moreover, the maximum traction due to interfacial cavitation isindependent of the water thickness (Fig. 6b), which defines an interfacial strength forthe wet adhesion (i.e., pull-out stress). We note that the magnitude of the maximum traction depends on the interactions between water and graphene, or equivalently, the hydrophobicity of graphene. On the other hand, the corresponding separation at the maximum traction depends on the initial water thickness 
(Fig. 6b), which may be predicted by setting $\sigma=\sigma_{m}$ in Eq. (7). As discussed earlier, for relatively thick water films $\left(h_{0} \geq 4 \mathrm{~nm}\right)$, this separation increases linearly with $h_{0}$.

\subsection{Stage II: cavitation growth}

As observed in MD simulations, the growth of cavitationin the water film depends on the initial thickness $h_{0}$. For a relatively thick water film $\left(h_{0}=4 \mathrm{~nm}\right)$, the cavity is nearly hemispherical (Fig. 7 a-c), maintaining a $90^{\circ}$ contact angle with graphene. The radius of the hemispherical cavity can be related to the surface tension of water $(\gamma)$ and the average traction $(\sigma)$ by force balance (Young-Laplace equation):

$$
R=\frac{2 \gamma}{\sigma},
$$

As the cavity radius increases, the traction $\sigma$ decreases. The initial radius of the cavity is set by the maximum traction: $R_{0}=2 \gamma / \sigma_{m}$, which defines a minimum thickness for the water film with a hemispherical cavity. In other words, if $h_{0}<R_{0}$, the cavity cannot take the hemispherical shape, as discussed later.

The growth of ahemispherical cavity is limited by the size of the periodic box in the MD simulations, i.e., $R \leq L / 2$, which sets a lower bound for the traction in Stage II:

$$
\sigma_{I I}=\frac{4 \gamma}{L} .
$$

When $R=L / 2$, the neighboring cavities come into contact andcoalesce, entering the next stage. The transition however often occurs when the neighboring cavities are sufficiently close but before contacting. Apparently, the size of the periodic box is arbitrarily set in the MD simulations, with no physical significance. A more realisticscenorio may be that multiple cavities 
are nucleated over a large area and they grow independently until coalescence. The phenomenon is thus more complicated, beyond the scope of the present study. Nevertheless, we focus on the traction-separation behavior due to cavitation growth assuming a fixed box size $L$.

Neglecting the volume change of water as well as deformation of graphene, the separation $d$ can be related to the radius of the hemispherical cavity, which combines with Eq. (8) to yielda simple traction-separation relation for Stage II:

$$
d(\sigma)=\frac{16 \pi \gamma^{3}}{3 L^{2} \sigma^{3}}
$$

where $\sigma_{m} \geq \sigma \geq \sigma_{I I}$. Thus, the maximum separation in Stage II is: $d\left(\sigma_{I I}\right)=\frac{\pi L}{12}$. We note that the simple scaling, $d \sim \sigma^{-3}$, is a result of the constant volume assumption, without taking into account the thermodynamics of evaporation/condensation [57]. However, the effect of water volume change could be significant at high stresses. For example, at $\sigma=\sigma_{m}$, the volumetric strain of water is $5-10 \%$ for the water films (Fig. 6a). Moreover, the deflection of the graphene monolayer could also be important in the present case. Without a detailed analysis of these effects, Eq. (10) serves as a rough estimate of the traction-separation relation in Stage II.

To compare with MD simulations, we take $\gamma=0.0608 \mathrm{~N} / \mathrm{m}$ for the surface tension of water (discussed later).Figure 7d compares the cavity radius from MD simulations with Eq. (8), and Figure 7e compares the traction-separation relation (for $h_{0}=4 \mathrm{~nm}$ ) with Eq. (10). Overall the continuum analysis captures the qualitative trend for the cavitation growth and the tractionseparation behavior in MD simulations (Stage II). The quantitative agreement could be improved by taking into account the volume change of water and the deflection of graphene in a more detailed continuum analysis. 

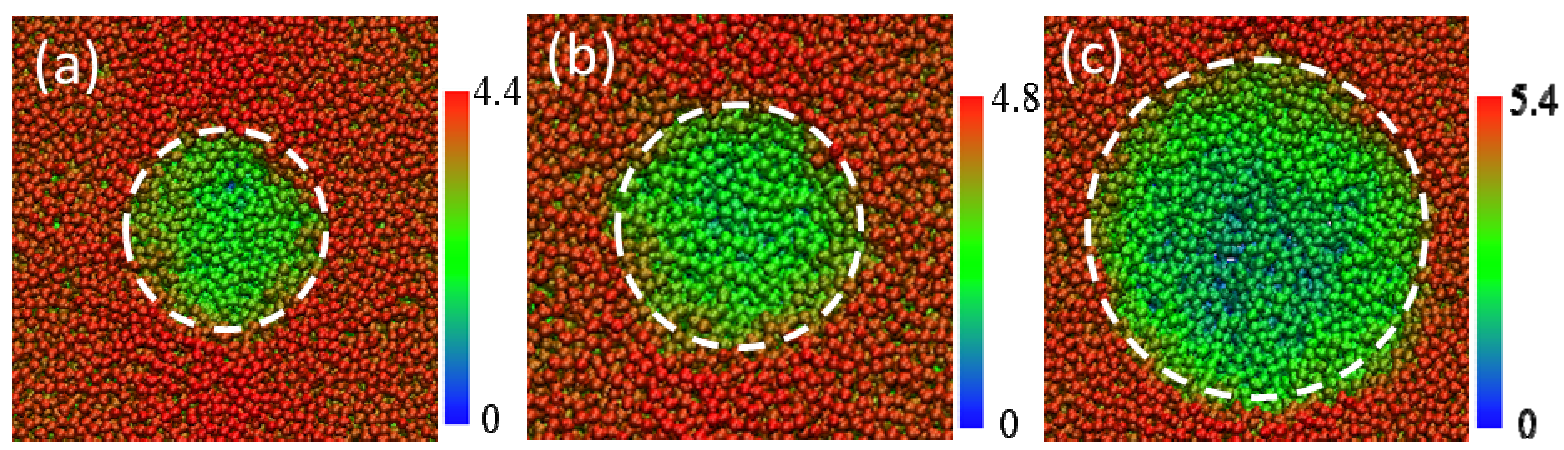

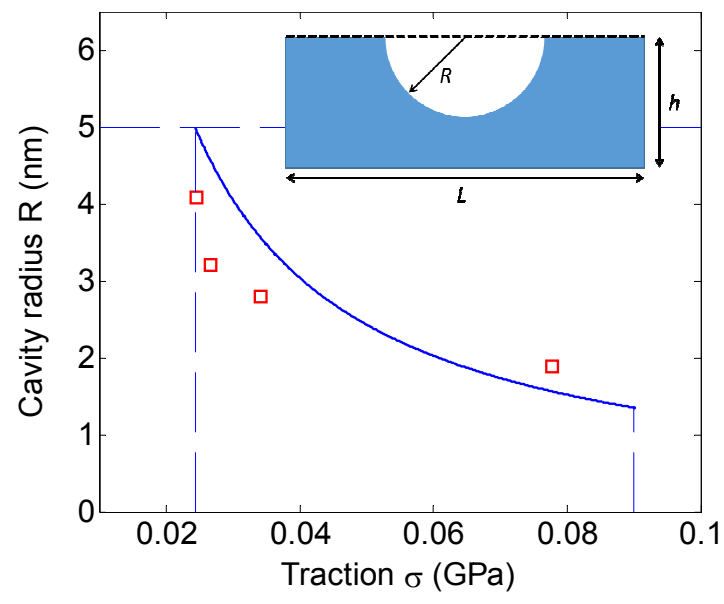

(d)

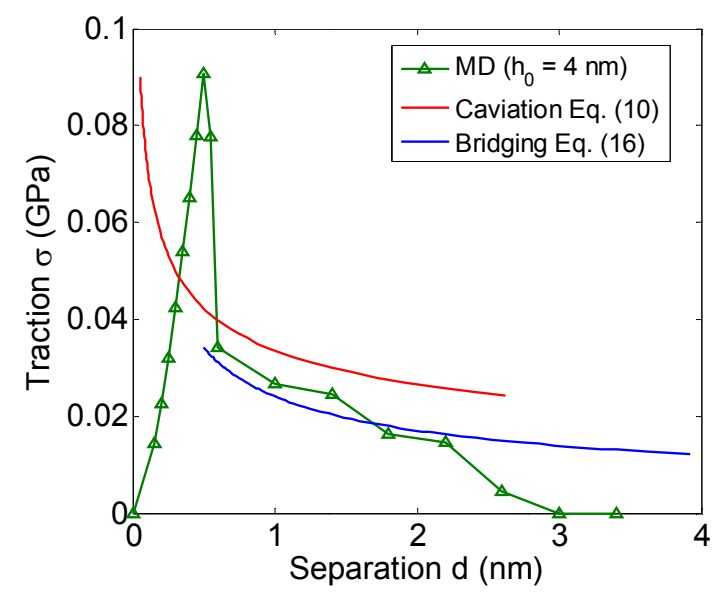

(e)

Figure 7. Growth of a hemispherical cavity in a relatively thick water film ( $\left.h_{0}=4 \mathrm{~nm}\right)$. (a-c) Snapshots of cavitation by MD simulations (top view, $\sigma=77.7,34.2$, and $24.6 \mathrm{MPa}$ ), showing water molecules colored by height contour (in $\mathrm{nm}$ ). The nearly circular top of the cavity gives a measure of the radius, shown in (d) along with the prediction by Eq. (8). The inset in (d) shows schematicside view ofa hemispherical cavity. (e) Traction-separation relation by MD in comparison with Eq. (10) for cavitation and Eq. (16) for capillary bridging.

By Eq. (8), taking $\sigma=\sigma_{m} \sim 90 \mathrm{MPa}$ and $\gamma=0.0608 \mathrm{~N} / \mathrm{m}$, the initiation radius of a hemispherical cavity is estimated to be $R_{0}=1.35 \mathrm{~nm}$. If the initial thickness of the water film is smaller than the initiation radius (i.e., $h_{0}<R_{0}$ ), a non-spherical through-thickness cavity forms and grows, as observed in MD simulations for $h_{0}=1 \mathrm{~nm}$ (see Fig. 8a-c). In this case, the 
cavitation growth can be analyzed approximately by assuming an axisymmetric cavity (see inset of Fig. 8d), governed by a couple of nonlinear differential equations:

$$
\left\{\begin{array}{c}
\frac{d \varphi}{d z} \sin \varphi+\frac{\sin \varphi}{r}=\frac{\sigma_{w}}{\gamma} \\
\frac{d r}{d z} \sin \varphi=\cos \varphi
\end{array}\right.
$$

where $\sigma_{w}$ is the hydrostatic tension in water and the angle $\varphi$ is a function of $z$ with boundary conditions set by the contact angles: $\varphi=0$ on the bottom surface $(z=0)$ and $\varphi=\pi / 2$ on the top surface $(z=h)$. The first equation in Eq. (11) is theYoung-Laplace equation for the axisymmetric cavity, and the other equation is the geometric relation for the surface profile of the cavity, $r(z)$. In addition, assuming no volume change of water, we have

$$
\int_{0}^{h} \pi r^{2} d z=L^{2} d
$$

where $h=h_{0}+d$.For a given water thickness $\left(h_{0}<R_{0}\right)$ and tension $\left(\sigma_{w}<\sigma_{m}\right)$, the surface profile $r(z)$ and the corresponding separation $d$ can be determined by an iterative procedure that numerically integrates Eq. (11) subject to the constraint in Eq. (12). The average traction acting on graphene is then, $\sigma=\sigma_{w}\left(1-\frac{\pi R_{0}^{2}}{L^{2}}\right)$, where $R_{0}=r(z=0)$ is the radius of substrate exposure.Figure $8 \mathrm{~d}$ showsthe surface profiles of the cavity at different traction levels for $h_{0}=1$ $\mathrm{nm}$, and Figure 8e shows thecorresponding traction-separation relation.Starting at the maximum traction of $90 \mathrm{MPa}$, a through-thickness cavity is formed. As the cavity grows, the stress decreases and the separation increases until the radius of the cavity at the top reaches $L / 2(5 \mathrm{~nm})$ with a traction of 26.4 MPa. In comparison with MD simulations, the continuum analysis again qualitatively captures the traction-separation behavior in Stage II. However, the through- 
thickness cavity in the MD simulation does not take an axisymmetric shape in general, possibly due to the heterogeneous surface structures of the a- $\mathrm{SiO}_{2}$ substrate.

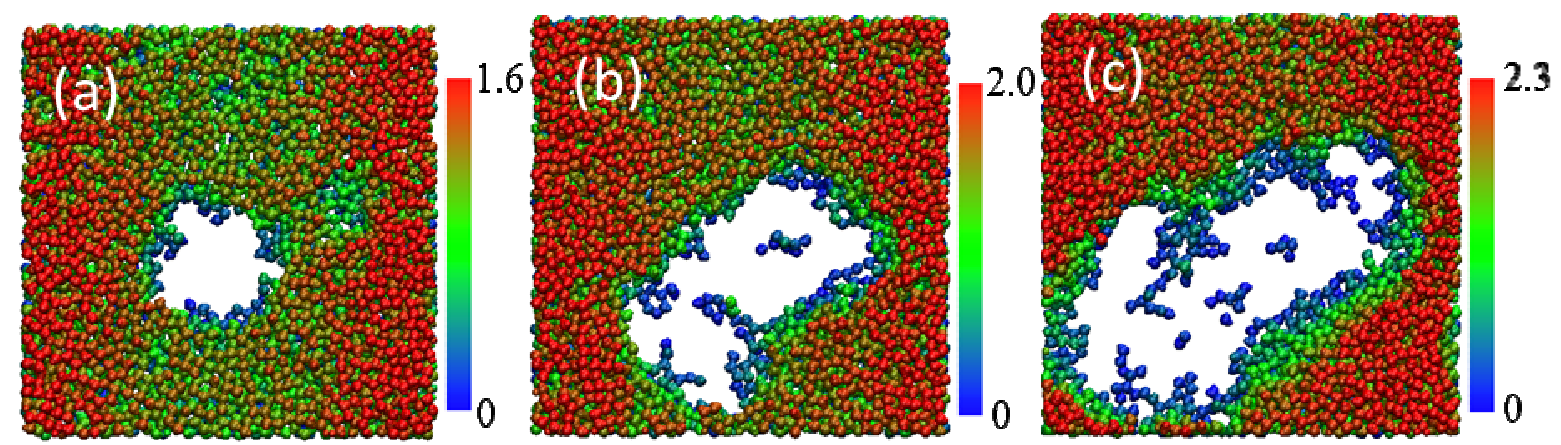

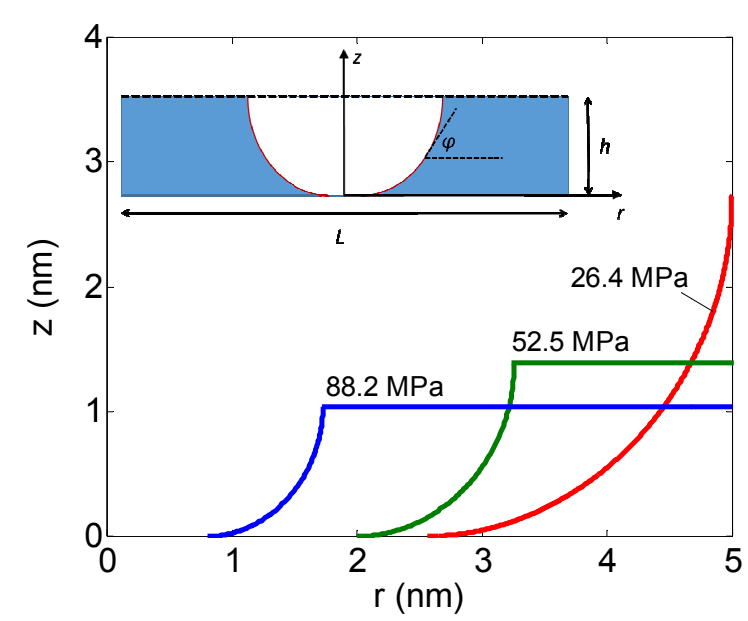

(d)

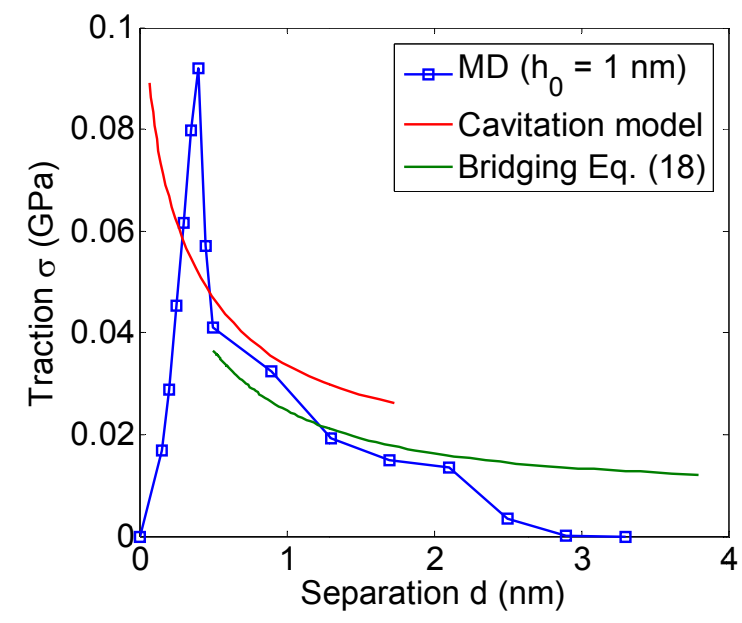

(e)

Figure 8: Growth of a through-thickness cavity in a thin water film ( $\left.h_{0}=1 \mathrm{~nm}\right)$. (a-c) Snapshots of cavitation by MD simulations (top view, $\sigma=57.1,32.5$, and 19.2 $\mathrm{MPa}$ ), showing water molecules colored by height contour (in $\mathrm{nm}$ ) and exposure of the substrate surface.(d) Axisymmetric cavitation growth by a continuum analysis, showing the profiles corresponding to $\sigma=88.2,52.5$, and 26.4 MPa. The inset shows the schematic side view of the cavitation model.(e) Traction-separation relation by MD in comparison with the axisymmetric cavitation model and Eq. (17) for the capillary bridging model.

The MD simulation for thecase with a water film of thickness $h_{0}=2.5 \mathrm{~nm}$ shows yet another scenario of cavitation growth:a nearly hemispherical cavity forms first, which grows and reaches the bottom of the water film, exposing the substrate surface (see Fig. 9).Again, assuming 
no volume change of the water, when the hemispherical cavity reaches the substrate surface, we have

$$
L^{2}\left(R-h_{0}\right)=\frac{2}{3} \pi R^{3}
$$

For $R_{0} \leq R \leq L / 2$, Eq. (13) can be satisfied only if

$$
R_{0} \leq h_{0} \leq \frac{2 L}{3 \sqrt{2 \pi}}
$$

which defines a range of intermediate thickness for the water film. With $R_{0}=1.35 \mathrm{~nm}$ and $L=10$ $\mathrm{nm}$, this range is between $1.35 \mathrm{~nm}$ and $2.66 \mathrm{~nm}$. Indeed, the MD simulation with $h_{0}=2.5 \mathrm{~nm}$ is in this range, whereas the case with $h_{0}=4 \mathrm{~nm}$ (Fig. 7) is not and thus no substrate exposure. It may be expected that the corresponding traction-separation relation in this intermediate range lies in between of the previous two cases (i.e., hemispherical and through-thickness cavitation). For $h_{0}$ $=2.5 \mathrm{~nm}$, a continuum analysis predicts a traction-separation relation very close to the case of hemispherical cavitation [48]. Similar to Fig. 7e, the continuum analysis qualitatively captures the traction-separation behavior in Stage II.
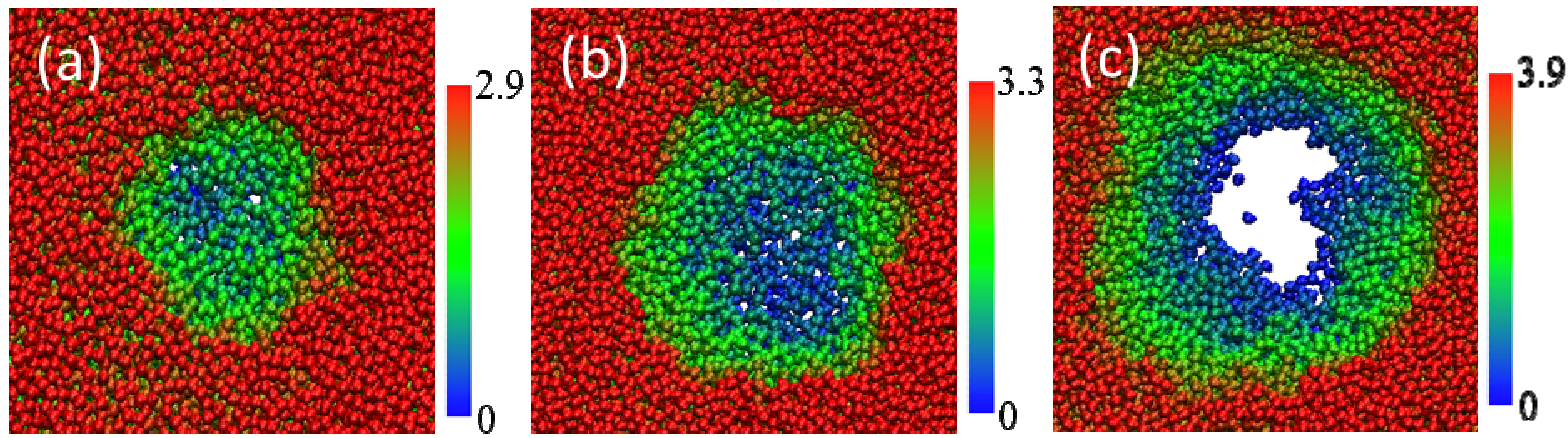

Figure 9. Cavitation in a water film of intermediate thickness $\left(h_{0}=2.5 \mathrm{~nm}\right)$ by MD simulations. (a-c) Plan-view snapshots for $\sigma=78.5,26.7$, and $21.8 \mathrm{MPa}$, showing water molecules colored by height contour (in $\mathrm{nm}$ ) and exposure of the substrate surface in (c). 


\subsection{Stage III: Capillary bridging}

Cavitation growthin Stage II eventually leads to coalescence of neighboring cavities and the formation ofcapillary bridges. As shown in Figure 10, by replicating the periodic boxin the MD simulations, we seethe morphological evolution of the capillary bridging in Stage III, from parallel ridgesto circular islands. For the case of a thin water film $\left(h_{0}=1 \mathrm{~nm}\right)$, the throughthickness cavities coalesce to form parallel ridges and islands with partially exposed substrate surface. For a relatively thick water film $\left(h_{0}=4 \mathrm{~nm}\right)$, the ridges and islands form on top of a continuous water layer covering the substrate surface. For the case of an intermediate thickness

$\left(h_{0}=2.5 \mathrm{~nm}\right)$, the morphology is more complicated with an array of circular holes at the bottom of the water layer, along the ridges and in between of the circular islands. The morphological evolution of capillary bridging could influence the traction-separation behavior, although the MD results for the three cases in Fig. 4 are similar. It is possible to study the capillary bridging by numerical simulations based on continuum models $[58,59]$. In the present study, we consider two simple models, one for parallel water ridges and the other for circular islands. 

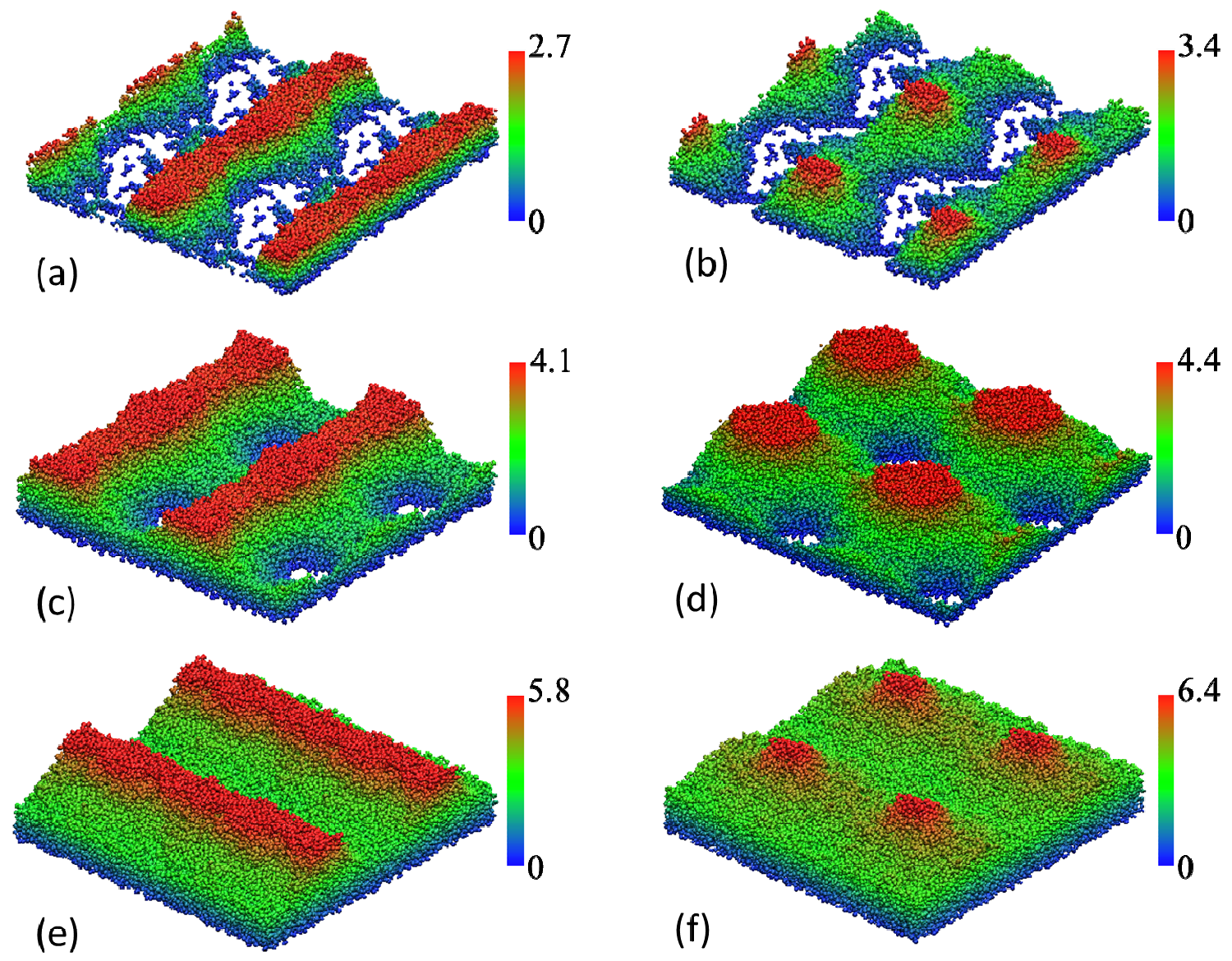

Figure 10. Morphological evolution of capillary bridging between graphene and a- $\mathrm{SiO}_{2}$ from $\mathrm{MD}$ simulations ( 2 by 2 periodic replication). (a-b) $h_{0}=1 \mathrm{~nm}, \sigma=13.43$ and $3.54 \mathrm{MPa}$; (c-d) $h_{0}=2.5$ $\mathrm{nm}, \sigma=17.56$ and $9.56 \mathrm{MPa}$; (e-f) $h_{0}=4 \mathrm{~nm}, \sigma=14.51$ and $4.59 \mathrm{MPa}$.

\section{Bridging withwater ridges}

The water bridging morphology in Fig. 10e can be described as parallel ridgeson top of a blanket layer of water. Figure 11 shows a cross-sectional view, where the side faces of the water ridge are approximately cylindrical with a radius $R$ andthe width of the ridge top is: $b=L-2 R$. The force balance requires that

$$
R=\frac{\gamma}{\sigma} .
$$


With $\sigma=16.2 \mathrm{MPa}, b=2.5 \mathrm{~nm}$ and $L=10 \mathrm{~nm}$ in Fig. 11, the surface tension of water can be determined by Eq. (15), giving $\gamma=0.0608 \mathrm{~N} / \mathrm{m}$. This value is in reasonable agreement with the typical value for surface tension of water $(\sim 0.07 \mathrm{~N} / \mathrm{m})$ and is used in all the continuum analyses of the present study for comparison with the MD simulations.

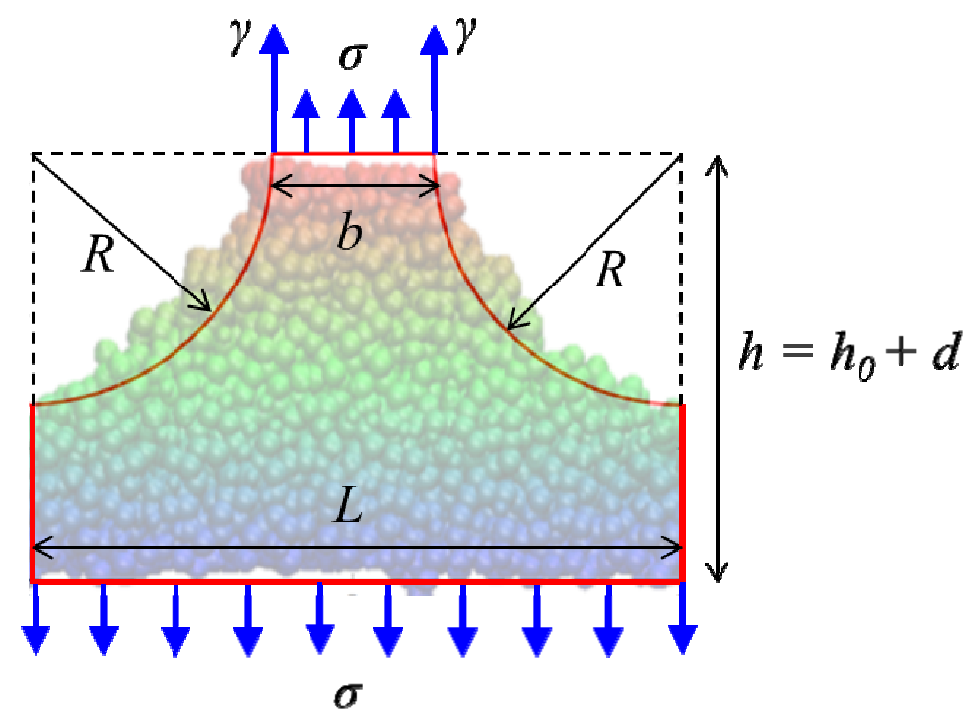

Figure 11: Cross section of a water ridge bridging between graphene and a- $\mathrm{SiO}_{2}$, comparing the continuum model (lines) with MD simulation $\left(h_{0}=4 \mathrm{~nm}\right.$ and $\left.\sigma=16.2 \mathrm{MPa}\right)$.

Again, by assuming no change of the water volume and neglecting the deflection of graphene,the separation is obtained as a function of the traction as

$$
d(\sigma)=\frac{\pi R^{2}}{2 L}=\frac{\pi \gamma^{2}}{2 L \sigma^{2}}
$$

Figure 11 compares the cross section of the water ridge from MD simulation with the continuum model $(R=3.75 \mathrm{~nm}$ and $d=2.21 \mathrm{~nm})$. As the separation $d$ increases, the traction $\sigma$ decreases with a scaling relation, $d \sim \sigma^{-2}$; note that this scaling is different from that in Eq. (10) for cavitation growth in Stage II. Eventually, when $R=L / 2$, the top of the ridge shrinks to a line $(b \rightarrow 0)$, 
setting a lower limit for the traction, $\sigma_{I I I}=\frac{2 \gamma}{L}$, and the corresponding limit separation $d\left(\sigma_{I I I}\right)=\frac{\pi L}{8}$.The traction-separation relation by this continuum model is shown in Fig. 7e in comparison with MD simulationfor the case with $h_{0}=4 \mathrm{~nm}$.Parallel ridges are observed in the MD simulation when the separation is around $2 \mathrm{~nm}$. Since the traction in this stage is relatively low $(<20 \mathrm{MPa})$, the effects of water volume change and graphene deflection are less significant so that the continuum model is fairly accurate. However, the parallel water ridges become unstable before the top width shrinks to zero, transitioning to water islands as shown in Fig. 10f. This transition is similar to break-up of a liquid jet as a result of the Rayleigh instability [60, 61], but with additional complexity due to the water-graphene interactions in the present case.

For the case of a thin water film (e.g., $h_{0}=1 \mathrm{~nm}$ ), the parallel water ridges become isolated with exposed substrate surface in between (Fig. 10a). For such isolated ridges, the average traction is related to the radius of the side face as

$$
\sigma=\frac{\gamma(b+2 R)}{R L}
$$

where $b=\frac{L h_{0}}{R}-\left(2-\frac{\pi}{2}\right) R$ (assuming no volume change of water). Thetraction-separation relation is then

$$
d(\sigma)=R-h_{0}=\sqrt{\frac{2 \gamma L h_{0}}{2 \sigma L-\pi \gamma}}-h_{0} .
$$

where the traction has a lower bound $\sigma_{I I I}=\frac{2 \gamma}{L}$ corresponding to $b=0$. As shown in Fig. 8e, Eq. (18) agrees with the MD results reasonably well in Stage III with 1-2 nm separation. Again, as 
the separation increases further, the parallel ridgesbecome unstable and break up into islands (Fig. 10b).

\section{Bridging with water islands}

In the last stage of the separation process, the parallelwater ridges break up to form water islands, similar to Rayleigh instability $[60,61]$. While the water islands appear to be circular at the top interface, the morphology is more complicated in general (see Fig. $10 \mathrm{~b}$, d and f). An approximate continuum analysis assuming an array of axisymmetric water islands on a continuous water filmwas performed [48]. However, under the constraint of the periodic box in MD simulations, no meaningful solution could be found for the cases considered in the present study $\left(h_{0}=1-4 \mathrm{~nm}\right.$ and $\left.L=10 \mathrm{~nm}\right)$.A more sophisticated continuum analysis is necessary to capture the morphological evolution of the water bridging during this stage. Nevertheless, the results from the MD simulations (see Fig. 4) suggest thatgraphene is fully separated from the substrate soon after the formation of water islands.

\section{Summary}

MD simulations are conducted to study the traction-separation behaviors for wet adhesion between graphene and $\mathrm{a}-\mathrm{SiO}_{2}$. Three stages of the traction-separation relationsare identified and they are analyzed approximately by simple continuum models. The work of separation (per unit area) is found to be close to the theoretical value $\left(60.0 \mathrm{~mJ} / \mathrm{m}^{2}\right)$ dictated by the interaction potential between graphene and water. The maximum traction is found to be set by the critical stress for cavitation at the water/graphene interface (around $90 \mathrm{MPa}$ in the present study). With morphological evolution of water from cavitation to capillary bridging, the range of interaction 
extends to about $3 \mathrm{~nm}$ before complete separation of graphene. Compared tovan der Waals interactionsfor dry adhesion between graphene and a- $\mathrm{SiO}_{2}$, the work of separation for wet adhesion is considerably smaller, the maximum traction is lower, but the range of interaction is longer. It is noted that the properties of wet adhesion depend sensitively on the graphene-water interactions, which may vary considerably between hydrophobic and hydrophilic interactions.

\section{Acknowledgments}

The authors gratefully acknowledge financial support of this work by the National Science Foundation through Grant No. CMMI-1130261. The authors acknowledge the Texas Advanced Computing Center (TACC) at the University of Texas at Austin for providing HPC resources that have contributed to the research results reported within this paper. 


\section{References}

[1] X. S. Li, Y. W. Zhu, W. W. Cai, M. Borysiak, B. Y. Han, D. Chen, R. D. Piner, L. Colombo, R. S. Ruoff, Transfer of Large-Area Graphene Films for High-Performance Transparent Conductive Electrodes, Nano Letters, 9: 4359-4363, 2009.

[2] S. Bae, H. Kim, Y. Lee, X. Xu, J. Park, Y. Zheng, J. Balakrishnan, T. Lei, H. R. Kim, Y. Song, Y. Kim, K. Kim, B. Ozyilmaz, J. Ahn, B. H. Hong and S. Lijima, Roll-to-roll production of 30-inch graphene films for transparent electrodes. Nature Nanotechnology, 5:574-578,2010.

[3] Y. M. Lin, C. Dimitrakopoulos, K. A. Jenkins, D. B. Farmer, H. Y. Chiu, A. Grill, P. Avouris, $100 \mathrm{GHz}$ transistors from wafer-scale epitaxial graphene, Science, 327: 662, 2010.

[4] S.R. Na, J.W. Suk, L. Tao, D. Akinwande, R.S. Ruoff, R. Huang, K. M. Liechti, Selective mechanical transfer of graphene from seed copper foil using rate effects, ACS Nano, 9:1325-1335, 2015.

[5] R. Huang, Graphene: Show of adhesive strength, Nature Nanotechnology, 6:537-538, 2011.

[6] Z. Lu and M. L. Dunn, van der Waals adhesion of graphene membrane, Journal of Applied Physics, 107:044301, 2010.

[7] T. Li and Z. Zhang, Substrate-regulated morphology of graphene, Journal of Physics DApplied Physics, 43: 075303, 2010.

[8] Z. H. Aitken and R. Huang, Effects of mismatch strain and substrate surface corrugation on morphology of supported monolayer graphene, Journal of Applied Physics, 107: 123531, 2010.

[9] W. Gao, P. Xiao, G. Henkelman, K. M. Liechti, and R. Huang, Interfacial adhesion between graphene and silicon dioxide by density functional theory with van der Waals corrections, Journal of Physics D: Applied Physics, 47: 255301, 2014.

[10] Z. Zong, C. L. Chen, M. R. Dokmeci, and K. T. Wan, Direct measurement of graphene adhesion on silicon surface by intercalation of nanoparticles, Journal of Applied Physics, 107: 026104, 2010.

[11] S. P. Koenig, N. G. Boddeti, M. L. Dunn, and J. S. Bunch, Ultrastrong adhesion of graphene membranes, Nature nanotechnology, 6: 543-546, 2011.

[12] N. G. Boddeti, S. P. Koenig, R. Long, J. L. Xiao, J. S. Bunch, and M. L. Dunn, Mechanics of Adhered, Pressurized Graphene Blisters, Journal of Applied Mechanics, 80: 040909, 2013.

[13] T. Yoon,W. C. Shin, T. Y. Kim, J. H. Mun, T. S. Kim, B. J. Cho, Direct Measurement of Adhesion Energy of Monolayer Graphene as-Grown on Copper and Its Application to Renewable Transfer Process, Nano Letter, 12:1448-1452, 2012.

[14] Z. Cao, P. Wang, W. Gao, L. Tao, J. W. Suk, R. S. Ruoff, et al., A blister test for interfacial adhesion of large-scale transferred graphene, Carbon, 69: 390-400, 2014.

[15] S. R. Na, J. W. Suk, R. S. Ruoff, R. Huang, and K. M. Liechti, Ultra Long-Range Interactions between Large Area Graphene and Silicon, ACS Nano, 8: 11234-11242, 2014. 
[16] A. W. Adamson and A. P. Gast, Physical Chemistry of Surfaces. Interscience publishers, New York, 1967.

[17] Y. W. Chen and H. P. Cheng, Structure and stability of thin water films on quartz surfaces, Applied Physics Letters, 97: 161909, 2010.

[18] J. J. Yang and E. G. Wang, Water adsorption on hydroxylated alpha-quartz (0001) surfaces: From monomer to flat bilayer, Physical Review B, 73: 035406, 2006.

[19] M. J. Lee, J. S. Choi, J. S. Kim, I. S. Byun, D. H. Lee, S. Ryu, C. Lee and B. H. Park, Characteristics and effects of diffused water between graphene and a $\mathrm{SiO} 2$ substrate, Nano Research, 5: 710-717, 2012.

[20] S. Wang, Y. Zhang, N. Abidi, L. Cabrales, Wettability and surface free energy of graphene films, Langmuir, 25: 11078-11081, 2009.

[21] Y. J. Shin, Y. Wang, H. Huang, G. Kalon, A. T. S. Wee, Z. Shen, C.S. Bhatia, H. Yang, Surface-energy engineering of graphene, Langmuir, 26: 3798-3802, 2010.

[22] K.-S. Kim, Hee-Jung Lee, C. Lee, S.-K. Lee, H. Jang, J.-H. Ahn, J.-H. Kim and H. J. Lee, Chemical vapor deposition-grown graphene: The thinnest solid lubricant, ACS Nano, 5: 5107-5114, 2011.

[23] R. Raj, S.C. Maroo, E.N. Wang, Wettability of graphene. Nano Letter, 13: 1509-1515, 2013.

[24] F. Taherian, V. Marcon, N.F.A. van der Vegt, F. Leroy, What is the contact angle of water on graphene. Langmuir, 29: 1457-1465, 2013.

[25] Z. Li, Y. Wang, A. Kozbial, G. Shenoy, F. Zhou, R. McGinley, P. Ireland, B. Morganstein, A. Kunkel, S.P. Surwade, L. Li, H. Liu, Effect of airborne contaminants on the wettability of supported graphene and graphite, Nature Materials, 12: 925-931, 2013.

[26] A. Kozbial, Z. Li, C. Conaway, R. McGinley, S. Dhingra, V. Vahdat, F. Zhou, B. D’Urso, H. Liu, L. Li, Study on the surface energy of graphene by contact angle measurements, Langmuir, 30: 8598-8606, 2014.

[27] J. Dong, Z. Yao, T. Yang, L. Jiang, and C. Shen, Control of superhydrophilic and superhydrophobic graphene interface, Scientific Reports, 3: 1733, 2013.

[28] J. Rafiee, X. Mi, H. Gullapalli, A. V. Thomas, F. Yavari, Y. Shi, P.M. Ajayan, N.A. Koratkar, Wetting transparency of graphene, Nature Materials, 11: 217-222, 2012.

[29] C.-J. Shih, Q.H. Wang, S. Lin, K.-C. Park, Z. Jin, M.S. Strano, D. Blankschtein, Breakdown in the wetting transparency of graphene. Physical Review Letter, 109: 176101, 2012.

[30] S. Plimpton, Fast parallel algorithms for short-range molecular dynamics, Journal of Computational Physics, 117: 1-19, 1995.

[31] D. W. Brenner, O. A. Shenderova, J. A. Harrison, S. J. Stuart, B. Ni, and S. B. Sinnott, A second-generation reactive empirical bond order (REBO) potential energy expression for hydrocarbons, Journal of Physics: Condensed Matter, 14: 783-802, 2002.

[32] M. Arroyo and T. Belytschko, Finite crystal elasticity of carbon nanotubes based on the exponential Cauchy-Born rule,Physical Review B, 69: 115415, 2004.

[33] Y. Huang, J. Wu and K. C. Hwang, Thickness of graphene and single-wall carbon nanotubes. Physical Review B 74: 245413, 2006. 
[34] Q. Lu and R. Huang, Nonlinear Mechanics of Single-Atomic-Layer Graphene Sheets, International Journal of Applied Mechanics, 1: 443-467, 2009.

[35] Q. Lu, W. Gao, and R. Huang, Atomistic simulation and continuum modeling of graphene nanoribbons under uniaxial tension, Modelling and Simulation in Materials Science and Engineering, 19: 054006, 2011.

[36] W. Gao and R. Huang, Thermomechanics of monolayer graphene: Rippling, thermal expansion and elasticity, Journal of the Mechanics and Physics of Solids, 66: 42-58, 2014.

[37] J. Tersoff, Modeling Solid-State Chemistry - Interatomic Potentials for Multicomponent Systems, Physical Review B, 39: 5566, 1989.

[38] S. Munetoh, T. Motooka, K. Moriguchi, and A. Shintani, Interatomic potential for Si-O systems using Tersoff parameterization, Computational Materials Science, 39: 334-339, 2007.

[39] H. J. C. Berendsen, J. R. Grigera, and T. P. Straatsma, The Missing Term in Effective Pair Potentials, Journal of Physical Chemistry, 91: 6269-6271, 1987.

[40] J.-P. Ryckaert, G. Ciccotti, and H. J. Berendsen, Numerical integration of the Cartesian equations of motion of a system with constraints: molecular dynamics of n-alkanes, Journal of Computational Physics, 23: 327-341, 1977.

[41] R. W. Hockney and J. W. Eastwood, Computer simulation using particles: CRC Press, 2010.

[42] C. T. Chen, R. A. Fine, and F. J. Millero, The equation of state of pure water determined from sound speeds, The Journal of Chemical Physics, 66: 2142-2144, 1977.

[43] Q. Zheng, D. J. Durben, G. H. Wolf, and C. A. Angell, Liquids at Large Negative Pressures: Water at the Homogeneous Nucleation Limit, Science, 254: 829-832, 1991.

[44] E. Herbert, S. Balibar, and F. Caupin, Cavitation pressure in water, Physical Review E, 74: $041603,2006$.

[45] F. Caupin, Liquid-vapor interface, cavitation, and the phase diagram of water, Physical Review E, 71: 051605, 2005.

[46] J. Ma, A. Michaelides, D. Alfe, L. Schimka, G. Kresse, and E. G. Wang, Adsorption and diffusion of water on graphene from first principles, Physical Review B, 84: 033402, 2011.

[47] T. Werder, J. H. Walther, R. L. Jaffe, T. Halicioglu, and P. Koumoutsakos, On the watercarbon interaction for use in molecular dynamics simulations of graphite and carbon nanotubes, Journal of Physical Chemistry B, 107: 1345-1352, 2003.

[48] W. Gao, Thermomechanical and interfacial properties of monolayer graphene. $\mathrm{PhD}$ Dissertation, The University of Texas at Austin, 2014.

[49] E. R. Cruz-Chu, A. Aksimentiev, and K. Schulten, Water-silica force field for simulating nanodevices, Journal of Physical Chemistry B, 110: 21497-21508, 2006.

[50] P. E. M. Lopes, V. Murashov, M. Tazi, E. Demchuk, and A. D. MacKerell, Development of an empirical force field for silica. Application to the quartz-water interface, Journal of Physical Chemistry B, 110: 2782-2792, 2006.

[51] N. T. Cuong, M. Otani, and S. Okada, Semiconducting Electronic Property of Graphene Adsorbed on (0001) Surfaces of SiO2, Physical Review Letters, 106: 106801, 2011.

[52] X. F. Fan, W. T. Zheng, V. Chihaia, Z. X. Shen, and J. L. Kuo, Interaction between graphene and the surface of SiO2, Journal of Physics-Condensed Matter, 24: 305004, 2012. 
[53] D. A. Litton and S. H. Garofalini, Modeling of hydrophilic wafer bonding by molecular dynamics simulations, Journal of Applied Physics, 89: 6013-6023, 2001.

[54] E. Paek and G. S. Hwang, A computational analysis of graphene adhesion on amorphous silica, Journal of Applied Physics, 113: 164901, 2013.

[55] S. Cheng and M.O. Robbins, Capillary adhesion at the nanometer scale,Physical Review E, 89:062402, 2014.

[56] S. Timoshenko and S. Woinowsky, Theory of plates and shells. McGraw-Hill, New York, 1959.

[57] H.-J. Butt and M. Kappl, Normal capillary forces, Advances in Colloid and Interface Science, 146: 48-60, 2009.

[58] F.M. Orr, L.E. Scriven and A.P. Rivas, Pendular rings between solids: meniscus properties and capillary force, Journal of Fluid Mechanics, 67: 723-742, 1975.

[59] J. Qian and H. Gao, Scaling effects of wet adhesion in biological attachment systems, Acta Biomaterialia 2: 51-58, 2006.

[60] L. Rayleigh, On the instability of jets, Proc. London Math. Soc. 10: 4-13, 1878.

[61] J. Eggers, Nonlinear dynamics and breakup of free surfaces flows, Reviewsof Modern Physics,3: 865-929,1997. 